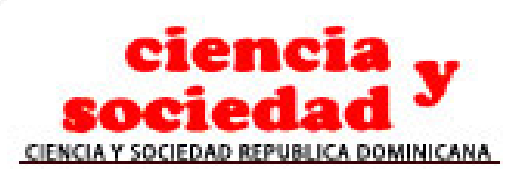

Ciencia y Sociedad

ISSN: 0378-7680

dpc@mail.intec.edu.do

Instituto Tecnológico de Santo Domingo

República Dominicana

Hernández Madrigal, Mónica; Ramírez Flores, Élfego

EVALUACIÓN DE PROYECTOS DE INVERSIÓN CON BENEFICIOS INTANGIBLES: APLICACIÓN

DEL MODELO COSTO - EFECTIVIDAD EN LA PREVENCIÓN DEL CÁNCER CERVICOUTERINO

Ciencia y Sociedad, vol. 38, núm. 1, 2013, pp. 115-139

Instituto Tecnológico de Santo Domingo

Santo Domingo, República Dominicana

Disponible en: http://www.redalyc.org/articulo.oa?id=87026374006

Cómo citar el artículo

Número completo

- Más información del artículo

Página de la revista en redalyc.org

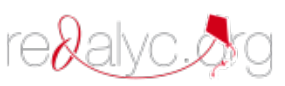

Sistema de Información Científica

Red de Revistas Científicas de América Latina, el Caribe, España y Portugal

Proyecto académico sin fines de lucro, desarrollado bajo la iniciativa de acceso abierto 


\section{EVALUACIÓN DE PROYECTOS DE INVERSIÓN CON BENEFICIOS INTANGIBLES: APLICACIÓN DEL MODELO COSTO - EFECTIVIDAD EN LA PREVENCIÓN DEL CÁNCER CERVICOUTERINO}

Assessment of Investment Projects with Intangible Benefits: An Application of the Cost-Effectiveness Model in the Cervical Cancer Prevention

\section{Mónica Hernández Madrigal Élfego Ramírez Flores}

Resumen: El objetivo del presente trabajo consiste en analizar el modelo costo-efectividad como medida para evaluar los beneficios intangibles de un proyecto de salud pública relacionado con la prevención del cáncer cervicouterino. Tomando como punto de partida la situación actual de la evolución del cáncer cervical, se identifican dos métodos esenciales para la evaluación económica de los proyectos de salud: el análisis costo-beneficio y el análisis costoefectividad. Los resultados enfatizan la importancia de la utilización de índices basados no solo en términos monetarios, sino también en medidas no monetarias. De esta forma, se sientan las bases para la utilización de medidas intangibles en la evaluación de proyectos de inversión, por medio del desarrollo de índices tales como calidad, satisfacción, salud, riesgo, percepción y aprendizaje.

Palabras claves: Beneficios intangibles, costo-efectividad, costobeneficio, cáncer cervicouterino. 
Abstract: The aim of this paper is to analyze the cost-effectiveness model as a measure to assess the intangible benefits of a public health project related to the prevention of cervical cancer. Taking as starting point the current situation of cervical cancer evolution, we identify two essential methods for economic evaluation of health projects: the cost-benefit and cost-effectiveness models. The results highlight the relevance of the use of indexes based not only in monetary terms but also in non-monetary measures. This research promotes the use of intangible measures in the evaluation of investment projects through the development of indexes such as quality, satisfaction, health, risk perception and learning.

Keywords: Intangible benefits, cost-effectiveness, cost-benefit, cervical cancer.

\section{Introducción}

El cáncer cervicouterino $(\mathrm{CaCu})$ se encuentra entre las primeras causas de muerte en mujeres de todos los grupos de edad (Flores et al., 2002; IMSS, 2009). Según datos del Banco Mundial, en 2001 se estimaron un total de 26,000 muertes relacionadas con esta enfermedad en Latinoamérica. Específicamente, según la Organización Mundial de la Salud (OMS, 2010), el CaCu está considerado como el tercer cáncer más frecuente en mujeres a nivel mundial, y el segundo más frecuente entre mujeres de 15 a 44 años. En este sentido, los resultados obtenidos a partir de un estudio realizado por el Banco Mundial (Brown et al., 2006) señalan que el cáncer de mama, el cervical, el estomacal, el pulmonar y el de colon se encuentran entre los cinco primeros tipos de cáncer que afectan a las mujeres. En el caso de México, entre las principales causas de mortalidad para las mujeres se pueden encontrar el tumor maligno del cuello del útero, de mama, estómago, hígado y vías biliares intrahepáticas, así como el de 
Evaluación de proyectos de inversión con beneficios intangibles: aplicación del modelo costo - efectividad en la prevención del cáncer cervicouterino

tráquea, bronquios y pulmón (INEGI, 2010). De tal modo, la muerte de una mujer no solo tiene repercusiones en su círculo familiar, sino también que "representa una gran pérdida de años de vida productiva que contribuye al deterioro económico del país y a la ruptura de su tejido social" (SSA, 2002: 19).

De acuerdo con varios estudios, existe evidencia significativa de que dicho cáncer afecta en mayor medida a países en vías de desarrollo que a países desarrollados como consecuencia de los siguientes factores: ausencia de una infraestructura de salud pública bien desarrollada, baja calidad de las dietas consumidas en dichos países, escaso acceso a la información, bajos niveles de mayor educación, inequidad de género, acceso limitado a los servicios, pobreza y ausencia de métodos de prevención básicos y servicios de detección oportuna (Flores et al., 2002; SSA, 2002; OPS, 2004). Cerca del $86 \%$ de los casos de $\mathrm{CaCu}$ ocurren en países en vías de desarrollo, representando el 13\% de los cánceres femeninos (OMS, 2010). El gráfico $\mathrm{N} .^{\circ} 1$ presenta el número anual de nuevos casos de $\mathrm{CaCu}$ por grupo de edades en regiones desarrolladas y en vías de desarrollo.

\section{Gráfico N. ${ }^{\circ} 1$}

Número de nuevos casos de cáncer cervicouterino por grupo de edades (2008)

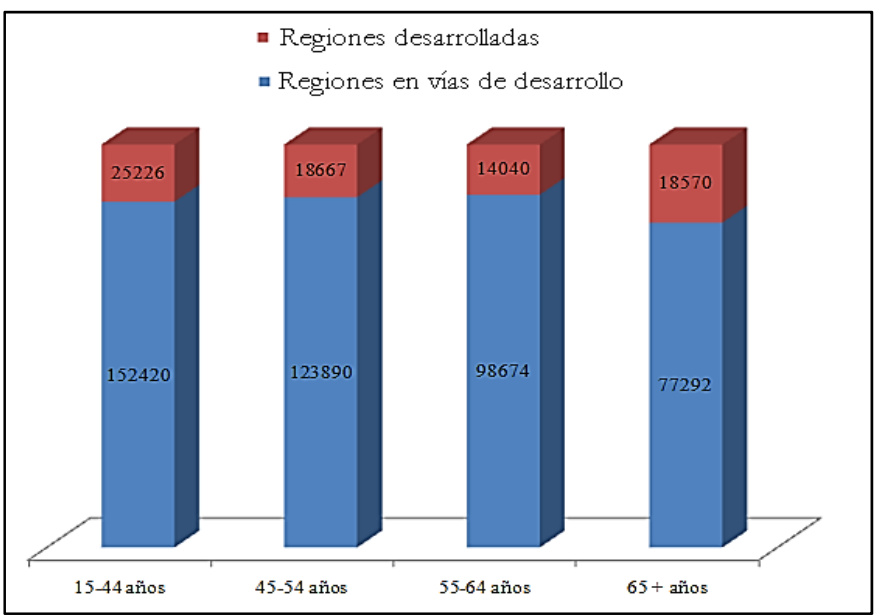

Fuente: OMS (2010). 
Según estadísticas de la Organización Mundial de Salud (OMS, 2010), el mundo tiene una población de 2,337 millones de mujeres en edades comprendidas de 15 años en adelante. Cada año 529,409 mujeres son diagnosticadas con $\mathrm{CaCu}$ y 274,883 mueren de dicha enfermedad. Así, mientras la tabla N. 1 muestra las estadísticas mundiales clave relacionadas con el desarrollo del $\mathrm{CaCu}$ por el año 2008, el gráfico N. ${ }^{\circ} 2$ muestra la comparación entre la tasa de incidencia y mortalidad por regiones del mundo, obtenidas a partir del estudio de la OMS en el año 2008. Para dicho Organismo, las tasas de mortalidad por $\mathrm{CaCu}$ son sustancialmente más bajas que las tasas de incidencia con un ratio de mortalidad a incidencia de 52\% (OMS, 2010).

\section{Tabla N. ${ }^{\circ} 1$}

Estadísticas mundiales del cáncer cervicouterino

\begin{tabular}{|l|c|c|c|}
\hline \multicolumn{1}{|c|}{ Población } & Mundo & $\begin{array}{c}\text { Regiones } \\
\text { en vías de } \\
\text { desarrollo }\end{array}$ & $\begin{array}{c}\text { Regiones } \\
\text { desarrolladas }\end{array}$ \\
\hline $\begin{array}{l}\text { Mujeres en riesgo de CaCu } \\
\text { (población femenina, edades } \\
>=15 \text { años) en miles }\end{array}$ & $2,336,986$ & $1,811,867$ & 525,120 \\
\hline Carga del cáncer cervicouterino & & & 76,507 \\
\hline $\begin{array}{l}\text { Número anual de nuevos casos } \\
\text { de CaCu }\end{array}$ & 529,409 & 452,902 & 33,159 \\
\hline $\begin{array}{l}\text { Número anual de muertes por } \\
\text { CaCu }\end{array}$ & 274,883 & 241,724 & 81,868 \\
\hline $\begin{array}{l}\text { Número proyectado de nuevos } \\
\text { casos de CaCu en 2025 }\end{array}$ & 719,385 & 668,120 & 38,291 \\
\hline $\begin{array}{l}\text { Número proyectado de muertes } \\
\text { por CaCu en 2025 }\end{array}$ & 394,699 & 380,212 & \\
\hline
\end{tabular}

Fuente: OMS (2010). 
Evaluación de proyectos de inversión con beneficios intangibles: aplicación del modelo costo - efectividad en la prevención del cáncer cervicouterino

Gráfico N. ${ }^{\circ} 2$

Tasa de incidencia y mortalidad por cáncer cervicouterino en el mundo (2008)

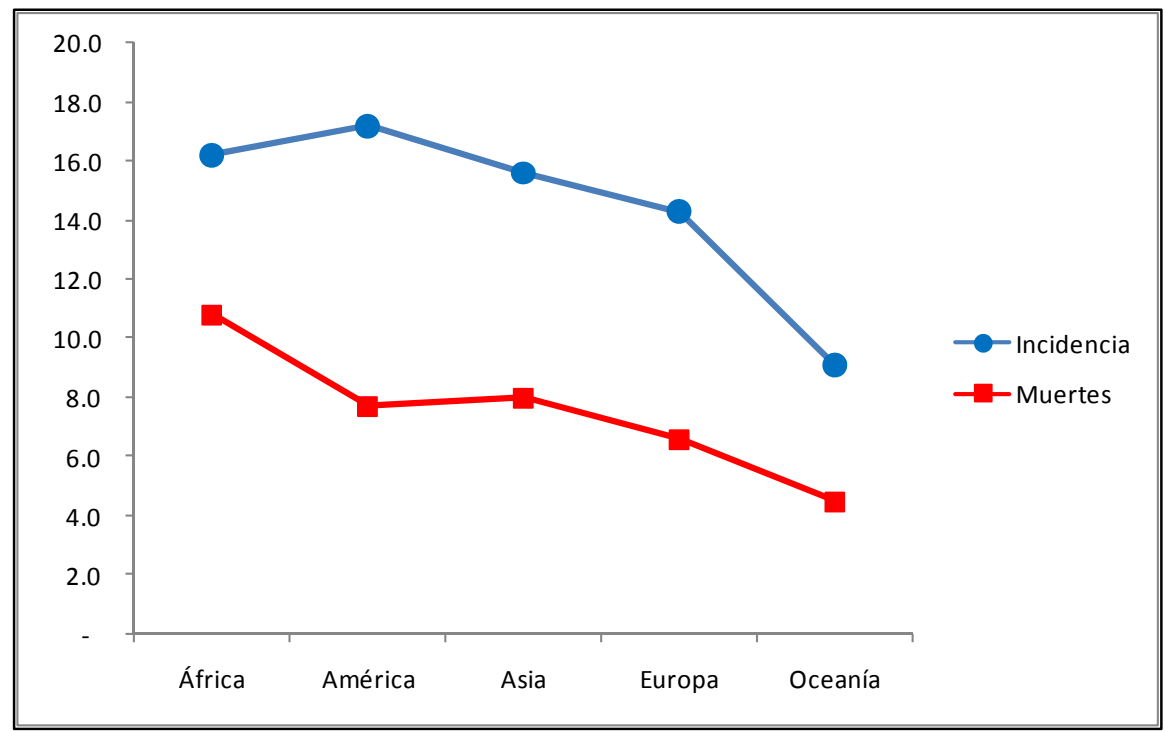

Fuente: OMS (2010).

Como se observa en el gráfico N. ${ }^{\circ}$ 2, las regiones de África y América muestran los niveles más altos tanto de incidencia como de muertes por $\mathrm{CaCu}$. Específicamente en el caso de América, de acuerdo con la Organización Panamericana de la Salud (OPS, 2004), durante los últimos cuarenta años, la mortalidad por $\mathrm{CaCu}$ y las tasas de incidencia en América Latina y el Caribe no han descendido tan significativamente como en América del Norte. Mientras que en Canadá y Estados Unidos se han observado tasas significativamente bajas, inferiores a 10 casos por cada 100,000 mujeres, en la mayoría de los países de América Latina y el Caribe las tasas anuales de $\mathrm{CaCu}$ se mantienen altas (OPS, 2004).

Por lo que respecta al caso de México, el cáncer ocupa el segundo lugar como causa de muerte en las mujeres (SSA, 2002) y, desde 1998 hasta 2005, el CaCu ocupó el primer lugar de causa de muerte por cáncer, siendo desplazado a partir de 2006 por el cáncer de 
mama (INEGI, 2010). De acuerdo con datos del INEGI (2010), desde 1998 se observa una disminución constante del $\mathrm{CaCu}$. El gráfico N. 3 presenta el porcentaje de defunciones por las cinco primeras causas de tumores malignos en las mujeres.

\section{Gráfico N.$^{\circ} 3$}

Porcentaje de defunciones en mujeres debidas a tumores malignos (1998-2008)

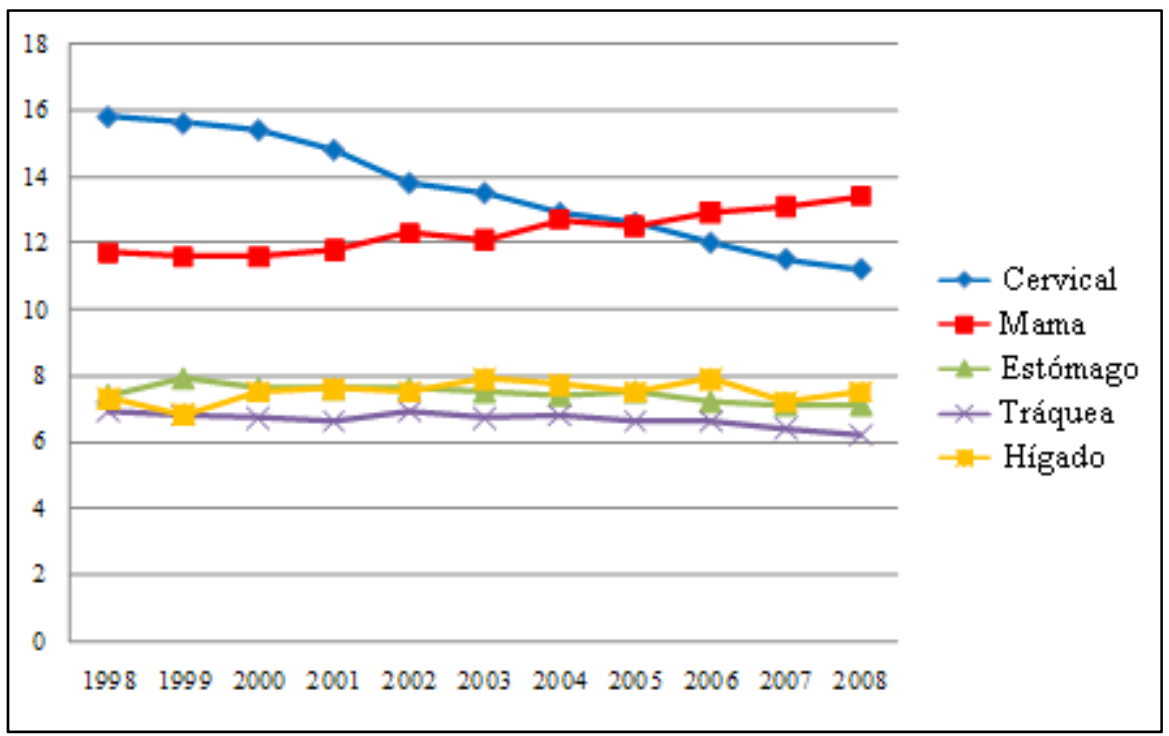

Fuente: INEGI (2010).

Sin embargo, si bien los casos de $\mathrm{CaCu}$ han disminuido con el tiempo, aún es alarmante la alta tasa de mortalidad de México, comparada con las tasas de los países desarrollados y de algunos países de Latinoamérica. Con base en datos del Instituto Mexicano del Seguro Social (IMSS), anualmente se detectan 15 mil nuevos casos de dicho cáncer, en cuyo caso, aproximadamente el 30\% pueden estar en peligro de perder la vida si la enfermedad no es diagnosticada tempranamente.

En lo que respecta a las tasas de morbilidad en México, medidas a través de los egresos hospitalarios por tumores malignos registrados 
Evaluación de proyectos de inversión con beneficios intangibles: aplicación del modelo costo - efectividad en la prevención del cáncer cervicouterino

durante el año 2000, fueron detectadas 125,950 muestras con alteraciones, de las cuales el $60.9 \%$ corresponden a displasia leve, el $18.5 \%$ a displasia moderada, el 8.6\% a displasia grave, el 6.5\% a cáncer in situ, y el $5.5 \%$ a cáncer invasor (SSA, 2002). Más recientemente, según datos del INEGI (2010), durante el periodo 2004-2007, si bien el cáncer de mama fue la principal causa de egreso hospitalario para las mujeres, en segundo lugar se ubican los egresos hospitalarios por tumores en el cuello del útero, que muestran una tendencia a la baja pasando de $15.2 \%$ a $11.7 \%$ en el mismo periodo. En 2007 , el $\mathrm{CaCu}$ es desplazado por las leucemias, las cuales mostraron un incremento en este año (INEGI, 2010).

Por tanto, como consecuencia de sus elevadas tasas de mortalidad y morbilidad, el $\mathrm{CaCu}$ representa uno de los principales problemas de salud pública en México (SSA, 2002). Sin embargo, el CaCu es una enfermedad prevenible. Según la Organización Panamericana de la Salud (OPS, 2004), el CaCu se puede prevenir y curar a un costo y riesgos bajos cuando el tamizaje para facilitar la detección oportuna de lesiones precursoras está disponible junto con el diagnóstico apropiado, el tratamiento y seguimiento. De tal modo, se hace evidente no solo la evaluación, el diagnóstico y el tratamiento de la enfermedad, sino también, y de forma más importante, la prevención de dicho cáncer ${ }^{1}$.

\section{Evaluación de proyectos de inversión: el modelo costo- beneficio y el modelo costo-efectividad}

La implementación de los métodos de prevención del $\mathrm{CaCu}$ a nivel de país supone costos para los gobiernos. De acuerdo con la OMS (2007), el costo representa la mayor barrera para hacer ampliamente disponible los programas de salud tendientes a la prevención y

Cabe destacar que en 1994, la Secretaría de Salud emitió la Norma Oficial Mexicana NOM-014-SSA2-1994 para la prevención, tratamiento y control de cáncer del cuello del útero y de la mama en la atención primaria. 
diagnóstico temprano ${ }^{2}$. En el caso de México, por ejemplo, debido a los altos costos, el gobierno negó la aprobación de incluir la aplicación de la vacuna del virus del papiloma humano (VPH) en el esquema de vacunación, exhortando mejor por el diagnóstico y detección del $\mathrm{CaCu}$ a través de pruebas de detección como la identificación de ADN de VPH y la prueba Pap (Cámara de Diputados, 2010). Al implementar técnicas de prevención, como por ejemplo la vacunación temprana, las instituciones de salud se enfrentan a decisiones importantes relacionadas con el financiamiento y los costos que dicho proyecto genera a los países.

La importancia de los costos y el financiamiento relacionados con proyectos sociales puede llegar a ejercer una fuerte presión en los gobiernos. Así, si bien es de esperarse que los beneficios de los programas de apoyo sean iguales para todos los miembros de la sociedad, a menudo los altos costos de dichos proyectos tienden a tomar medidas restrictivas, como la segregación de algunos sectores, que pueden frenar el desarrollo social de las comunidades. Sin embargo, de acuerdo con la Organización Mundial de la Salud (2007), un factor decisivo para el éxito de un programa de diagnóstico cervical consiste en el buen funcionamiento del programa como un todo, integrado con otros programas tales como salud del adolescente, la planificación familiar, la salud de la mujer, la maternidad sin riesgo, la inmunización y la prevención del VIH. Para dicho Organismo, los requerimientos incluyen la capacidad del programa de asegurar altos niveles de cobertura en la población objetivo, ofrecer alta calidad, y desarrollar y supervisar que los pacientes reciban un tratamiento apropiado y aceptable.

No obstante, dicha tarea no es fácil. Los recursos suelen ser escasos y los gobiernos se ven en la necesidad de demostrar que los sistemas públicos de salud aportan contribuciones medibles que mejoran la

2 En el año 2007, el precio de mercado de la vacuna, previo a los honorarios de los intermediarios, era de US $\$ 90$ la dosis. De tal modo, considerando las tres dosis previstas en el calendario de prevención, el costo de la vacunación es considerable, incluso para los países más desarrollados (OMS, 2007). 
Evaluación de proyectos de inversión con beneficios intangibles: aplicación del modelo costo - efectividad en la prevención del cáncer cervicouterino

salud de la población (Neumann et al., 2008). Además, el juicio sobre la importancia y la medición, el impacto y el alcance de los datos pueden afectar directa o indirectamente los resultados obtenidos.

Debido a las dificultades inherentes a la evaluación de los proyectos en materia de salud, es posible lograr un acercamiento al estudio de costos y beneficios que pueden generarse a partir del desarrollo de un proyecto de salud. De acuerdo con Neumann et al. (2008), varios investigadores han intentado medir la tasa de retorno de inversiones en programas de salud pública de varias formas, orientándose a los costos y beneficios, tal como un analista financiero calcularía la tasa de retorno de alternativas en un portafolio.

Con el objeto de determinar la factibilidad y viabilidad económica de un proyecto de salud a gran escala, se hace evidente la necesidad de aplicar teorías, herramientas y conceptos de economía a temas relacionados con la salud y el cuidado de la salud, lo cual se conoce como economía de la salud (health economics). La economía de la salud se relaciona con la asignación de recursos escasos con el fin de mejorar la salud (OHE, 2010), e incluye la realización de estudios de inversión, de análisis costo-beneficio y de análisis costo-efectividad que permitan identificar las oportunidades en la implementación de las nuevas técnicas de diagnóstico del $\mathrm{CaCu}$ que incluyan a toda la población.

Existen varios métodos de evaluación de proyectos de inversión. En los métodos basados en medidas monetarias, los beneficios y los costos son medidos en términos monetarios, y los proyectos son evaluados de forma individual. En contrapartida, en los métodos basados en medidas no monetarias, los beneficios se expresan en unidades intangibles como calidad, satisfacción, salud, riesgo, percepción y aprendizaje, y los proyectos son evaluados de forma comparativa.

En el contexto de la salud, se pueden identificar dos métodos esenciales para la valuación económica de los proyectos: el análisis 
costo-beneficio (monetario) y el análisis costo-efectividad (no monetario), el cual a su vez incluye diferentes modalidades de enfoque (figura N. ${ }^{\circ}$ 1) (Levin \& McEwan, 2001; Drummond et al., 2005).

Figura N. ${ }^{\circ} 1$

Métodos de valuación económica en proyectos sociales enfocados a la salud

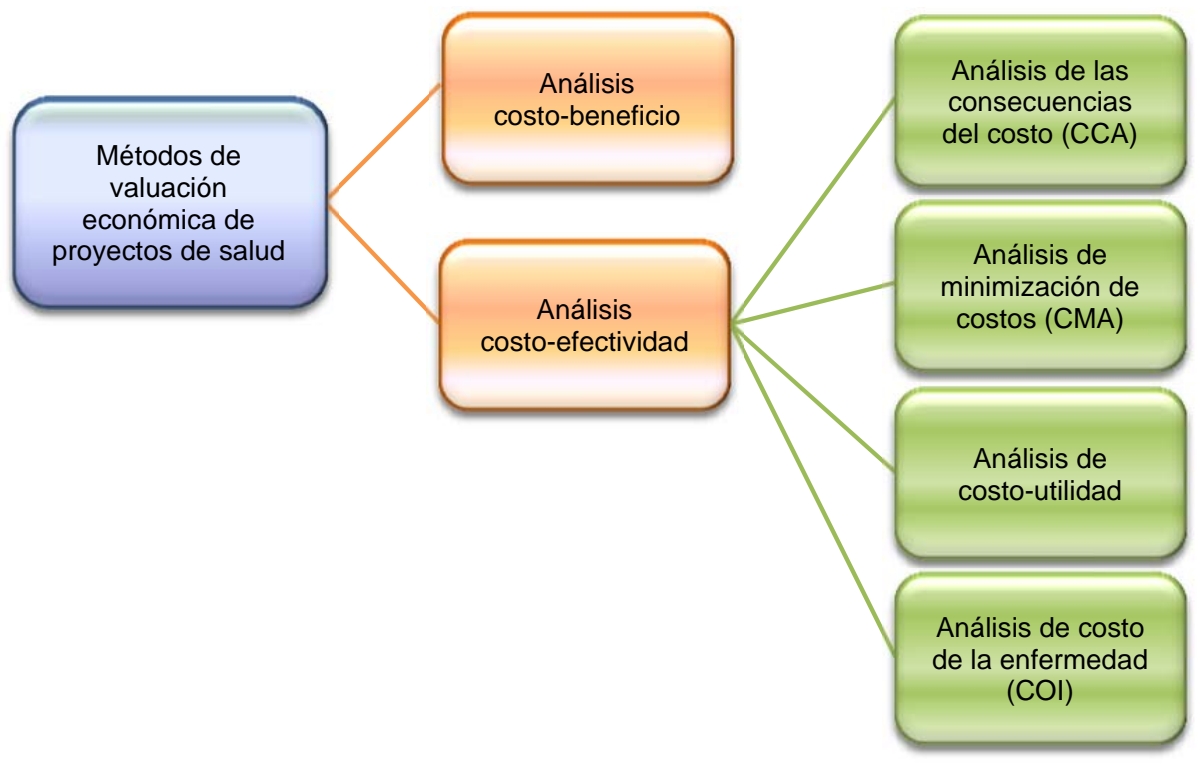

Fuente: Elaboración propia.

Por un lado, el análisis costo-beneficio (cost benefit analysis, CBA) es una forma de valuación económica en la cual los resultados y los costos son valuados en términos monetarios. El resultado del análisis será la diferencia o el cociente entre los beneficios y costos netos, expresados en términos monetarios (OHE, 2010; Academy Health, 2010). En dicho análisis, los analistas cuantifican los beneficios en salud ya sea desde una perspectiva de "capital humano" (midiendo el valor de la salud reducida como la pérdida de ganancias de los individuos afectados) o desde una perspectiva de "buena voluntad para pagar" (evaluando a través de datos de mercado o encuestas lo que la gente está dispuesta a pagar por algunos beneficios específicos de salud) (Neumann et al., 2008). 
Evaluación de proyectos de inversión con beneficios intangibles: aplicación del modelo costo - efectividad en la prevención del cáncer cervicouterino

Por otro lado, el análisis costo-efectividad de una intervención es una forma de valuación económica en la cual los costos deben estar justificados por los beneficios intangibles que ofrecen (OHE, 2010). Dicho método consiste en un análisis de los costos y las consecuencias incrementales en salud de una intervención, comparado con una alternativa. Los efectos o consecuencias (generalmente efectos de salud) de todos los programas comparados son medidos en unidades estandarizadas, como por ejemplo años de vida ajustados de calidad (QALY) ganados. El resultado de dicho análisis es una razón de costos incrementales al efecto incremental de una intervención (por ejemplo, costo por QALY) (Academy Health, 2010). Para Musgrove y Fox-Rushby (2006: 272), el análisis costo-efectividad "ofrece la manera más simple y clara de promover el valor por el dinero en salud". En palabras de dichos autores, dicha técnica "compara el costo de una actividad, llamada intervención, con los beneficios en salud conocidos o esperados" (p. 271).

Para realizar dicho análisis, existe una serie de métodos de valuación económica que pueden llevarse a cabo en el desarrollo de la investigación económica en el sector salud, los cuales comparan dos o más alternativas en términos de sus costos y beneficios (Tabla N. ${ }^{\circ}$ ).

La mayoría de los análisis costo-efectividad sobre técnicas de diagnóstico precoz del $\mathrm{CaCu}$ se han orientado a países de alto ingreso (Brown et al., 2006; Kulasingam \& Myers, 2003; Sanders \& Taira, 2003; Brisson et al., 2007; Bergeron et al., 2008; Chesson et al., 2008; Kulasingam et al., 2008; Annemans et al., 2009; Kim et al., 2009). Sin embargo, como resultado de la mayor atención otorgada por los organismos de salud a los países en desarrollo, en los últimos años se ha puesto de manifiesto el creciente interés en este tipo de estudios. Así, en los últimos años es posible encontrar evidencia empírica de estudios sobre prevención, detección y diagnóstico del $\mathrm{CaCu}$ realizados en países de ingreso bajo o medio (Flores et al., 2002; Suba et al., 2001; Goldie et al., 2001; Goldie et al., 2007; Insinga et al., 2007). 


\section{Tabla N. ${ }^{\circ} 2$}

\section{Modalidades del método de análisis costo-efectividad en proyectos sociales enfocados a la salud}

\begin{tabular}{|l|l|}
\hline \multicolumn{1}{|c|}{ Variable } & \multicolumn{1}{c|}{ Descripción } \\
\hline $\begin{array}{l}\text { Análisis de las conse- } \\
\text { cuencias del costo } \\
\text { (cost consequences } \\
\text { analysis, CCA) }\end{array}$ & $\begin{array}{l}\text { Una forma de análisis costo-efectividad que compara } \\
\text { intervenciones o programas alternativos en el cual } \\
\text { los componentes de los costos incrementales y las } \\
\text { consecuencias son calculados y enlistados, siendo } \\
\text { excluyentes ambas alternativas. }\end{array}$ \\
\hline $\begin{array}{l}\text { Análisis de minimi- } \\
\text { zación de costos } \\
\text { (costo minimisation } \\
\text { analysis, CMA) }\end{array}$ & $\begin{array}{l}\text { Una forma de valuación económica que compara } \\
\text { los costos de intervenciones alternativas las cuales } \\
\text { tienen efectos iguales. }\end{array}$ \\
\hline $\begin{array}{l}\text { Análisis costo } \\
\text { utilidad }\end{array}$ & $\begin{array}{l}\text { Una forma de análisis costo-efectividad en la cual } \\
\text { los resultados son expresados en términos de costo } \\
\text { por QALY ganado, con el objeto de incorporar la } \\
\text { prolongación y la calidad de vida. }\end{array}$ \\
\hline $\begin{array}{l}\text { Análisis del costo de } \\
\text { la enfermedad (cost of } \\
\text { illness analysis, COI) }\end{array}$ & $\begin{array}{l}\text { Una forma de análisis costo-efectividad que ofrece } \\
\text { una estimación monetaria del impacto económico } \\
\text { total (el cual incluye costos directos e indirectos) de } \\
\text { una enfermedad en particular o condición. }\end{array}$ \\
\hline
\end{tabular}

Fuente: Levin y McEwan (2001), Neumann et al. (2008), OHE (2010), Academy Health (2010).

\subsection{LA CUANTIFICACIÓN DE LOS BENEFICIOS EN MATERIA DE SALUD EN EL ANÁLISIS COSTO-EFECTIVIDAD}

De acuerdo con Musgrove y Fox-Rushby (2006: 272), "el análisis costo-efectividad y la carga de la enfermedad (disease burden) están relacionados porque la efectividad es la reducción de la carga causada por una intervención"3. La carga de enfermedad se define como el impacto de un problema de salud en un área, medida por los costos

3 En este caso, el diagnóstico molecular como prueba de tamizaje para la detección del cáncer cervicouterino. 
Evaluación de proyectos de inversión con beneficios intangibles: aplicación del modelo costo - efectividad en la prevención del cáncer cervicouterino

financieros, la mortalidad, la morbilidad y otros indicadores. En este contexto, los rendimientos obtenidos a partir de la reducción de la carga de la enfermedad pueden traducirse en dos beneficios específicos: reducción de la mortalidad, con base en la estimación de las muertes prevenidas, y la reducción de la morbilidad, es decir, de la proporción de personas que enferman en un sitio y tiempo determinado. Debido a que la toma de pruebas de diagnóstico precoz disminuyen la incidencia del $\mathrm{CaCu}$, se consideran como beneficios los costos ahorrados por tratamiento en caso de verrugas genitales y los costos por tratamiento en caso de cáncer (Insinga et al., 2007).

La carga de la enfermedad se cuantifica en términos de DALYs ${ }^{4}$ (disability-adjusted life years) o QALYs (quality-adjusted life years). Mientras que por un lado los DALYs miden la carga de la enfermedad expresada en el total de años perdidos como consecuencia de la enfermedad, la discapacidad o muerte prematura, por otro lado los QALYs miden la carga de la enfermedad (disease burden) indirectamente al calcular el número de años de vida, ajustados por calidad, que pueden ser agregados por los métodos de diagnóstico precoz. ${ }^{5}$ Dado que el presente estudio está enfocado en un diagnóstico de prevención del $\mathrm{CaCu}$, el cual pretende mejorar la calidad de vida de los pacientes ${ }^{6}$, fueron utilizados los QALYs como medida básica de beneficios obtenidos a partir de la reducción de la carga de la enfermedad. En la actualidad, los QALYs son utilizados en la mayoría de las evaluaciones económicas como parte del proceso de toma de decisiones (Sassi, 2006).

Los años de vida ajustados por calidad (quality-adjusted life years, QALY) representan una de las medidas más utilizadas para medir

4 La unidad de medida DALY fue introducida por el Banco Mundial y la OMS (Musgrove \& Fox-Rushby, 2006).

5 Los QALYs se miden de la siguiente forma: a cada año en perfecta salud se le asigna el valor de 1.0 y disminuye hasta 0.0 en caso de muerte. Si los años extras no se viven con plena salud, entonces a dichos años se les asigna un valor entre 0 y 1.

6 La calidad de vida se define como el bienestar subjetivo de una persona, el cual incluye dimensiones físicas, psicológicas y sociales (OHE, 2010). 
los beneficios de los programas de salud. El QALY constituyen una medida alternativa sobre lo que vale un año de vida si la persona sufre una o más limitaciones de varias clases y grados (Musgrove \& Fox-Rushby, 2006), la cual a su vez debe ser descontada en el futuro. ${ }^{7}$ Los QALYs capturan en una sola medida las ganancias por la reducción de la morbilidad y la mortalidad (Neumann et al., 2008). De tal modo, los beneficios obtenidos a partir de una mejor calidad de vida se traducirán no solo en menores tasas de mortalidad y morbilidad, sino también en una disminución del gasto público en materia de salud.

Otra medida utilizada para medir los beneficios de los programas de salud son los años de vida saludable ajustados (AVISA). Dicha medida fue desarrollada en México con el fin de estimar la carga nacional de las enfermedades (Rodríguez Ábrego et al., 2007). En palabras de Rodríguez Ábrego et al. (2007: 133), "la estimación de la carga de la enfermedad mediante los AVISA permite establecer prioridades en la atención de la salud no identificables con el solo uso de indicadores individuales tales como la morbilidad o la mortalidad. Asimismo, contribuye a identificar el perfil de salud de la población y los cambios en las principales causas de enfermedad y muerte que ocurren entre poblaciones en el curso del tiempo". Otro indicador que permite la medición de la carga de la enfermedad lo constituye la esperanza de vida saludable (EVISA), que mide el número equivalente de años de vida que se espera sean vividos con salud completa (Rodríguez Ábrego et al., 2007).

Además de los beneficios previamente señalados, las intervenciones en materia de diagnóstico precoz del $\mathrm{CaCu}$ producen también beneficios no relacionados con la salud. Para Musgrove y Fox-Rushby (2006), los beneficios no relacionados con la salud de las intervenciones producen otra clase de beneficios: mayor productividad mental y física de los beneficiados, posibilidad de continuar trabajando. No

Generalmente se utiliza un descuento al futuro del 3\% anual (Musgrove \& Fox-Rushby, 2006). 
Evaluación de proyectos de inversión con beneficios intangibles: aplicación del modelo costo - efectividad en la prevención del cáncer cervicouterino

obstante, en palabras de dichos autores, si bien dichos beneficios pueden ser descritos e identificados conceptualmente, su cuantificación conlleva cierta dificultad. La tabla N. ${ }^{\circ} 3$ muestra los beneficios que pueden obtenerse a partir del diagnóstico precoz del VPH.

\section{Tabla N. ${ }^{\circ} 3$}

\section{Beneficios obtenidos a partir del diagnóstico molecular para la detección del cáncer cervicouterino}

\begin{tabular}{|l|l|}
\hline \multicolumn{1}{|c|}{ Variable } & \multicolumn{1}{c|}{ Descripción } \\
\hline $\begin{array}{l}\text { Reducción de } \\
\text { mortalidad }\end{array}$ & $\begin{array}{l}\text { Estimación de las muertes prevenidas. Se utiliza la edad de } \\
\text { la muerte para obtener el número de años salvados, pero } \\
\text { descontados (por ejemplo, a la tasa del 3\% anual). Obtener } \\
\text { la edad en que se tomó la prueba. }\end{array}$ \\
\hline $\begin{array}{l}\text { Reducción de } \\
\text { morbilidad }\end{array}$ & $\begin{array}{l}\text { Costo por tratamiento en caso de verrugas genitales: \$2,000 } \\
\text { pesos; costo por tratamiento en caso de cáncer (Insinga, } \\
\text { 2007). }\end{array}$ \\
\hline $\begin{array}{l}\text { Beneficios no } \\
\text { relacionados } \\
\text { con la salud }\end{array}$ & $\begin{array}{l}\text { Si bien pueden ser descritos, son difíciles de cuantificar } \\
\text { (Musgrove \& Fox-Rushby, 2006). A menudo hacen que los } \\
\text { beneficiarios sean más productivos y puedan seguir con el } \\
\text { trabajo (Brown et al., 2006). }\end{array}$ \\
\hline
\end{tabular}

Fuente: Insinga, 2007; Musgrove \& Fox-Rushby, 2006; Brown et al., 2006.

\subsection{LA CUANTIFICACIÓN DE LOS COSTOS DE LOS PROGRAMAS DE SALUD EN EL ANÁLISIS COSTO-EFECTIVIDAD}

El análisis costo-efectividad se basa en la comparación de la estimación de los beneficios medidos en unidades naturales, físicas y empíricas contra el valor monetario de los costos de la intervención (Musgrove \& Fox-Rushby, 2006). En este caso, la intervención del diagnóstico molecular para la detección oportuna del $\mathrm{CaCu}$ estará justificada siempre y cuando los beneficios exceden a los de cualquier otra alternativa. Por tanto, la estimación de los costos de la intervención del diagnóstico molecular se constituye como el siguiente paso en el análisis costo-efectividad del proyecto. 
De acuerdo con la Secretaría de Salud (SSA, 2002), el costo institucional que representa la atención del $\mathrm{CaCu}$ está determinado por la oportunidad en su detección y prevención, el cual se reduce significativamente cuando más temprana es la intervención. En la tabla N. ${ }^{\circ} 4$ recogemos la clasificación de los distintos costos que pueden ser considerados en un análisis costo-efectividad.

\section{Tabla N. ${ }^{\circ} 4$}

Clasificación de los costos que pueden ser considerados en un análisis costo-efectividad

\begin{tabular}{|l|l|}
\hline \multicolumn{1}{|c|}{ Variable } & \multicolumn{2}{|c|}{ Descripción } \\
\hline Costos explícitos: \\
\hline CI & Costos de insumos \\
\hline CA & Costos administrativos \\
\hline CL & Costos de logística \\
\hline Cost/QALY & Costo por QALY (Insinga et al., 2007) \\
\hline Costos implícitos: \\
\hline CNM & $\begin{array}{l}\text { Costos no médicos: valor del tiempo necesario para viajar, } \\
\text { esperar y llevar a cabo las pruebas médicas y los procedi- } \\
\text { mientos; valor del tiempo dedicado al cuidado de los enfermos } \\
\text { o la pérdida de salarios de pacientes y de transporte (Insinga } \\
\text { et al., 2007). }\end{array}$ \\
\hline CI & $\begin{array}{l}\text { Costos indirectos para pacientes y sus familias (valor del } \\
\text { tiempo necesario para viajar, esperar y llevar a cabo las } \\
\text { pruebas médicas y procedimientos; valor del tiempo de los } \\
\text { cuidados a los enfermos) (Brown et al., 2006). }\end{array}$ \\
\hline Extern & $\begin{array}{l}\text { Externalidades o costos impuestos a terceras personas. } \\
\text { Generalmente son difíciles de estimar, por lo que se suelen } \\
\text { excluir del análisis (Brown et al., 2006). }\end{array}$ \\
\hline CINT & $\begin{array}{l}\text { Costos intangibles que incluyen el sufrimiento de los } \\
\text { pacientes (OHE, 2010). }\end{array}$ \\
\hline CO & $\begin{array}{l}\text { Costos de oportunidad: los recursos sacrificados por tomar } \\
\text { una alternativa en específico. }\end{array}$ \\
\hline
\end{tabular}


Evaluación de proyectos de inversión con beneficios intangibles: aplicación del modelo costo - efectividad en la prevención del cáncer cervicouterino

Para realizar el presente estudio, fueron distinguidos los costos en directos e indirectos (Brown et al., 2006; Musgrove \& Fox-Rushby, 2006). Los costos directos representan el valor de aquellos recursos directamente implicados en la detección del virus, tales como el tiempo destinado por el profesional para tomar la muestra, las medicinas, el equipo, los costos de pacientes (e. g. tiempo de desplazamiento y gastos) para recibir tratamiento (OHE, 2010), los costos de insumos (material de salud), los costos administrativos (papelería, mano de obra, capacitación del personal) y los costos de logística (incluidos aquellos que permiten hacer llegar las tomas a la población objetivo) (Brown et al., 2006).

Los costos indirectos miden el impacto de la enfermedad y tratamiento con base en el tiempo de trabajo (y la habilidad para trabajar) pagado y no pagado (costos de productividad perdida) (OHE, 2010). Se refieren a aquellos costos de difícil identificación, muchas veces considerados costos no médicos, los cuales afectan de alguna forma la calidad de vida de los pacientes y sus familiares. Entre este tipo de costos podemos mencionar el valor del tiempo necesario para viajar, esperar y llevar a cabo las pruebas médicas y los procedimientos, el valor del tiempo dedicado al cuidado de los enfermos o la pérdida de salarios de pacientes y de transporte (Musgrove \& Fox-Rushby, 2006; Insinga et al., 2007).

De igual forma, las externalidades o costos impuestos a terceras partes deben ser incluidos en los estudios costo-efectividad relacionados con el sector salud (Musgrove \& Fox-Rushby, 2006). Las externalidades existen cuando la acción de un individuo impone costos o no ofrece beneficios a otras partes no involucradas en la transacción, y que no son compensados por los efectos que pueden causarse (Coase, 1960; Steinacker, 2006). Sin embargo, debido a la dificultad en la estimación de dichos tipos de costos, la mayoría de los estudios relacionados con el análisis costo-efectividad en aspectos de salud tienden a excluir este tipo de costos (Musgrove \& Fox-Rushby, 2006; Insinga et al., 2007). Además, existen algunos costos intangibles, como el dolor y el sufrimiento que resulta de padecer un tratamiento, 
que si bien son raramente incluidos como un costo en la valuación económica, pueden tener algún efecto en las medidas de calidad de vida (OHE, 2010). Por último, es importante tomar en cuenta los costos de oportunidad, es decir, los recursos sacrificados para el logro de un objetivo calculados implícitamente porque no representan un flujo monetario explícito.

\section{Conclusiones}

La relevancia de evaluar de forma sistemática los costos y los beneficios de los diferentes métodos de prevención del $\mathrm{CaCu}$ queda demostrada con los datos epidemiológicos del impacto que a nivel mundial, nacional y regional, tiene el $\mathrm{CaCu}$, ya que según la OMS, con datos de 2010, dicho cáncer está considerado como el tercer tipo más frecuente en mujeres a nivel mundial (OMS, 2010).

Las estadísticas comparativas de la morbilidad y mortalidad del $\mathrm{CaCu}$ son significativamente diferentes entre regiones desarrolladas y en vías de desarrollo, con una proporción de incidencia de 7 a 1 . Específicamente, México tiene un reto enorme: por encontrarse en una región [de los países] en vías de desarrollo y por ser el tumor maligno de cuello de útero una de las principales causas de mortalidad entre las mujeres, tiene un reto enorme en la prevención, detección y tratamiento de este grave problema epidemiológico.

Una ventaja que presenta el $\mathrm{CaCu}$ es que, a diferencia del cáncer de mama, dicho cáncer sí es prevenible, lo cual implica que el desarrollo, prueba y evaluación de los métodos de detección oportuna se conviertan en políticas públicas de salud prioritarias, es decir, que pueden ser rentable para el gobierno invertir en este tipo de métodos de detección. Por tanto, el cálculo de los costos y beneficios de los métodos de detección resultan relevantes no solo como un tema de presupuesto, sino también en materia de bien común. 
Evaluación de proyectos de inversión con beneficios intangibles: aplicación del modelo costo - efectividad en la prevención del cáncer cervicouterino

Así, mientras que el modelo costo-beneficio limita sus resultados a términos monetarios, la evaluación del modelo costo-efectividad reconoce la importancia de los aspectos tantos monetarios como no monetarios, lo cual a su vez permite la construcción de índices de medición indirecta de los beneficios intangibles (por ejemplo, índice de satisfacción, índice de corrupción, índice de calidad, índice de años de vida restante con calidad, entre otros) que inciden directamente en proyectos sociales.

A pesar de las limitaciones y supuestos de ambos métodos de evaluación -costo beneficio y costo efectividad-, es trascendente el esfuerzo de calcular los costos y los beneficios, tanto para tener un fundamento que sirva como base para tomar la decisión sobre políticas públicas en materia de salud, como para refinar dichos cálculos en estudios subsecuentes. La problemática de salud del país exige que se estandaricen estas metodologías con el fin de resolver con rapidez los grandes retos de salud que tiene nuestro país y nuestro Estado. Este artículo permitió comparar dos métodos de evaluación conceptualmente diferentes como lo son el de costo beneficio y el de costo efectividad, lo cual permite una complementariedad que fortalece las conclusiones y sienta las bases para la utilización de otros métodos y para la identificación de las ventajas y limitaciones de cada uno de ellos, proporcionando una mayor fundamentación a la toma de decisiones en el campo de la salud. 


\section{Referencias}

Academy Health (2010). Glossary. Washington: Academy Health. Recuperado el 14 de octubre de 2010 de http://www. hsrmethods.org/Glossary/Terms/C/Cost $\%$ 20Effectiveness $\% 20$ Analysis.aspx.

Annemans, L., Rémy, V., Oyee, J. y Largeron, N. (2009). CostEffectiveness Evaluation of a Quadrivalent Human Papillomavirus Vaccine in Belgium. PharmacoEconomics, 27(3), 231-245.

Bergeron, C., Largeron, N., McAllister, R., Mathevet, P. y Remy, V. (2008). Cost-effectiveness Analysis of the Introduction of a Quadrivalent Human Papillomavirus Vaccine in France. International Journal of Technology Assessment in Health Care, 24(1), 10-19.

Brisson, M., Van de Velde, N., De Wals, P. \& Boily, M. C. (2007). The Potential Cost-Effectiveness of Prophylactic Puman Papillomavirus Vaccines in Canada. Vaccine, 25(29), 5399-5408.

Brown, M. L., Goldie, S. J., Draisma, G., Harford, J. \& Lipscomb, J. (2006). Health Service for Cancer Control in Developing Countries. En Disease Control Priorities in Developing Countries (pp. 569-589). New York: Oxford University Press, World Bank.

Cámara de Diputados (2010). De la Secretaría de Gobernación con el que remite contestación a punto de acuerdo aprobado por la Cámara de Diputados. Gaceta Parlamentaria, 2994-I. Recuperado el 18 de octubre de 2010 desde http://sil.gobernacion.gob. $\mathrm{mx} /$ Archivos/Documentos/2010/04/asun_2646388_201004 06_1270582896.pdf.

Coase, R. H. (1960). The Problem of Social Cost. Journal of Law and Economics, 3, pp. 1-44. 
Evaluación de proyectos de inversión con beneficios intangibles: aplicación del modelo costo - efectividad en la prevención del cáncer cervicouterino

Chesson, H. W., Ekwueme, D. U., Saraiya, M. y Markowitz, L. E. (2008). Cost-Effectiveness of Human Papilloma Virus Vaccination in the United States. Emerg Infect Dis, 14(2), 244-251.

Drummond, M. F., Torrance, G. W., O’Brien, B. J. \& Stoddart, G. L. (2005). Métodos para la evaluación económica de los programas de asistencia sanitaria. Oxford: Oxford University Press.

Flores, Y., Shah, K., Lazcano, E.; Hernández, M., Bishai, D., Ferris, D. G., Lorincz, A., Hernández, P., \& Salmerón, J. (2002). Design and Methods of the Evaluation of an HPV-Based Cervical Cancer Screening Strategy in Mexico: the Morelos HPV Study. Salud Pública de México, 44(4), 335-344.

Goldie, S. J., Kuhn, L., Denny, L., Pollack, A y Wright, T. C. (2001). Policy Analysis of Cervical Cancer Screening Strategies in LowResource Settings: Clinical Benefits and Cost-Effectiveness. Journal of the American Medical Association, 285(24), 3107-3115.

Goldie, S. J., Kim, J. J., Kobus, K., Goldhaber-Fiebert, J. D., Salomonb, J., O'Sheaa, M. K. H., Boschc, F. X., De Sanjoséc, S. y Franco, E. (2007). Cost-effectiveness of HPV 16, 18 vaccination in Brazil. V accine 25(33), 6257-6270.

Insinga, R. P., Dasbach, E. J., Elbasha, E. H., Puig, A. y ReynalesShigematsu, L. M. (2007). Cost-Effectiveness of Quadrivalent Human Papillomavirus (HPV) in Mexico: a Transmission Dynamic Model-based Evaluation. Vaccine, 26, 128-139.

Instituto Mexicano del Seguro Social (IMSS) (2009). Atiende el IMSS con cirugía laparoscópica a mujeres con cáncer. Comunicado. Coordinación de Comunicación Social, 70. Recuperado el 21 de septiembre de 2010 de http://www.imss.gob.mx/NR/ rdonlyres / 21287260-1682-48F1-9E93-55B1F3A90EC7/ 0/020309Com70.pdf. 
Instituto Nacional de Estadística y Geografía (INEGI). (2010). Estadísticas a propósito del Día Mundial contra el Cáncer. México: INEGI. Recuperado el 15 de octubre de 2010 de http://www. inegi.org.mx/inegi/contenidos/espanol/prensa/Contenidos/ estadisticas/2010/cancer10.doc.

Kim, J. J., Ortendahl, J., \& Goldie, S. J. (2009). Cost-effectiveness of Human Papillomavirus Vaccination and Cervical Cancer Screening in Women Older than 30 Years in the United States. Annals of Internal Medicine, 151(8), 538-545.

Kulasingam, S. L., y Myers, E. R. (2003). Potential health and economic impact of adding a human papillomavirus vaccine to screening programs. Journal of the American Medical Association, 290(6), 781-89.

Kulasingam, S., Benard, S., Barnabas, R., Largeron, N. y Myers, E. (2008). Adding a quadrivalent human papillomavirus vaccine to the UK cervical cancer screening programme: A costeffectiveness analysis. Cost Effectiveness \& Resource Allocation, 6, 1-11.

Levin, H. M. y McEwan, P. J. (2001). Cost-Effectiveness Analysis. Methods and Applications. California: Sage Publications.

Musgrove, P. y Fox-Rushby, J. (2006). Cost-effectiveness analysis for priority setting. En Disease Control Priorities in Developing Countries (p. 271-285). New York: Oxford University Press, World Bank.

Neumann, P. J., Jacobson, P. D. y Palmer, J. A. (2008). Measuring the value of public health systems: the disconnect between health economists and public health practitioners. American Journal of Public Health, 98(12), 2173-2180. 
Evaluación de proyectos de inversión con beneficios intangibles: aplicación del modelo costo - efectividad en la prevención del cáncer cervicouterino

Office of Health Economics (OHE). (2010). Glossary. Washington: OHE. Recuperado el 14 de Octubre de 2010 de http://www. ohe.org/page/glossary.cfm.

Organización Mundial de la Salud (OMS). (2002). Cervical cancer screening in developing countries: report of a WHO consultation. France: World Health Organization.

Organización Mundial de la Salud (OMS). (2007).Vaccinating Against Cervical Cancer. Bulletin of the World Health Organization, 85 (2). Ginebra: OMS. Recuperado el 21 de septiembre de 2010 de http:/ /www.who.int/bulletin/volumes/85/2/07-020207 /en/index.html.

Organización Mundial de la Salud. (2007). Estrategia mundial de prevención y control de las infecciones de transmisión sexual 2006-2015. Ginebra: OMS. Recuperado el 21 de septiembre de 2010 desde http://whqlibdoc.who.int/publications/2007/978924356347 3_spa.pdf.

Organización Mundial de la Salud (OMS). WHO/ICO Information Centre on HPV and Cervical Cancer (HPV Information Centre). (2010). Human Papillomavirus and Related Cancers in World. Summary Report 2010. Ginebra: OMS. Recuperado el 15 de octubre de 2010 de www.who.int/hpvcentre.

Organización Panamericana de la Salud (OPS). (2004). Análisis de la situación del cáncer cervicouterino en América Latina y el Caribe. Washington: Organización Panamericana de la Salud.

Rodríguez-Abrego, G., Escobedo de la Peña, J., Zurita, B., y Ramírez, T. J. (2007). Muerte prematura y discapacidad en los derechohabientes del Instituto Mexicano del Seguro Social. Salud Pública de México, 49(2), 132-143. 
Sanders, G. D. y Taira, A. V. (2003). Cost Effectiveness of a Potential Vaccine for Human Papillomavirus. Emerging Infectious Diseases, 9(1), 37-48.

Sassi, F. (2006). Calculating QALYs, Comparing QALY and DALY calculations. Health Policy and Planning, 21(5), 402-408.

Secretaría de Salud (SSA). (2002). Programa de Acción: Cáncer Cérvico Uterino. México: Secretaría de Salud. Recuperado el 15 de octubre de 2010 de http://taxco.ses-gro.gob.mx/pro-acc/cancer _cervico_uterino.pdf.

Secretaría de Salud (SSA). (2006). Mortalidad por Cáncer Cérvico Uterino según Entidad Federativa y Municipio, 2005. México: Secretaría de Salud.

Steinacker, A. (2006). Externalities, Prospect Theory, and Social Construction: when will Government Act, what will Government do? Social Science Quarterly, 87(3), 459-476.

Suba, E. J., Nguyen, C. H., Nguyen, B. D. y Raab, S. S. (Viet/ American Cervical Cancer Prevention Project). (2001). De Novo Establishment and Cost-Effectiveness of Papanicolaou Cytology Screening Services in the Socialist Republic of Vietnam. Cancer, 91(5), 928-939. 


\section{Mónica Hernández Madrigal}

Doctora en Metodologías y Líneas de Investigación, en Contabilidad y Auditoría por la Universidad de Cantabria, España. Profesora de tiempo completo de la Universidad Autónoma de San Luis Potosí en México, en cuya Facultad de Contaduría y Administración imparte las asignaturas de Administración de Costos y Contabilidad. Autora y coautora de artículos sobre contabilidad de gestión; ha participado como ponente en distintos congresos y reuniones de la especialidad.

Email: hmadrigalmonica@uaslp.mx

\section{Élfego Ramírez Flores}

Maestro en Administración por el Instituto Tecnológico de Estudios Superiores de Monterrey (ITESM). Profesor de tiempo completo de la Facultad de Contaduría y Administración de la Universidad Autónoma de San Luis Potosí, en México, donde imparte las asignaturas de Economía del Sector Público. Ha sido ponente invitado en distintos congresos y reuniones y ha impartido cursos y conferencias en distintas universidades del país.

Email: elfegorf@gmail.com 
